digital subtraction angiography. Patients had subsequently undergone percutaneous transluminal renal angioplasty (PTRA).

The gadolinium group $(n=57)$ had a lower incidence of contrast nephropathy (increase in serum creatinine of $44 \mu \mathrm{mol} / \mathrm{l}[0.5 \mathrm{mg} / \mathrm{dl}]$ within 7 days of PTRA) than did the iodinated contrast group ( $n=68)$ or the combination group $(n=38$; $5.3 \%$ vs $20.6 \%$ and $10.5 \%$, respectively). No patients who received gadolinium required permanent dialysis or transplantation within 30 days, compared with $7.2 \%$ of those who received iodinated contrast $(P<0.005)$. During long-term follow-up (mean $40 \pm 22$ months), there was no significant difference between the groups in the number of patients who required renal replacement therapy or in all-cause mortality. The technical success of PTRA was $>90 \%$ for all groups, indicating that each agent provided vascular imaging of sufficient quality. Two patients who received gadolinium developed nephrogenic systemic fibrosis.

The authors conclude that gadolinium contrast seems to be as effective as iodinated contrast and safer with respect to contrast nephropathy in patients with pre-existing renal dysfunction who undergo renal revascularization.

Original article Kane GC et al. (2008) Comparison between gadolinium and iodine contrast for percutaneous intervention in atherosclerotic renal artery stenosis: clinical outcomes. Nephrol Dial Transplant 23: 1233-1240

\section{Hemodialysis access surveillance does not improve access outcomes}

Observational studies suggest that routine measurement of access blood flow and use of Doppler ultrasound can detect subclinical stenosis in hemodialysis vascular accesses. There is, therefore, interest in evaluating whether such screening improves access outcomes in patients on hemodialysis. Tonelli et al. have systematically analyzed data from the relevant randomized, controlled trials.

The analysis included 12 trials conducted in 1,164 hemodialysis patients with arteriovenous grafts or fistulas. The studies compared access screening by ultrasound dilution (in patients with grafts or fistulas) or Doppler ultrasound (in patients with grafts) with standard care, during 6-30 months of follow-up (median 15 months). In patients with fistulas, surveillance decreased the risk of access thrombosis (relative risk [RR] 0.47, 95\% Cl 0.28-0.77; event rate 18\%; $n=360)$. The risk of fistula loss was not, however, significantly decreased by surveillance (RR 0.65, 95\% Cl 0.28-1.51; event rate 13\%; $n=141)$. There was no decrease with surveillance in either the risk of graft thrombosis (RR 0.94, 95\% Cl 0.77-1.16; event rate 43\%; $n=446)$ or the risk of graft loss (RR 1.08, 95\% Cl 0.83-1.40; event rate 35\%; $n=381$ ).

Given that hemodialysis vascular access screening by use of ultrasound does not seem to decrease the risk of access loss in patients with arteriovenous fistulas or grafts, the authors conclude that electing not to routinely carry out such screening is reasonable, although additional trials, in particular of fistula screening, are highly desirable.

Original article Tonelli M et al. (2008) Ultrasound monitoring to detect access stenosis in hemodialysis patients: a systematic review. Am J Kidney Dis 51: 630-640

\section{KDOQI PTH target associated with high risk of low-turnover bone disease in stage 5 CKD}

KDOQI guidelines recommend maintenance of serum intact parathyroid hormone (iPTH) levels at 150-300 ng/l. Barreto et al. evaluated the effectiveness of this target for preventing renal osteodystrophy in patients with stage 5 chronic kidney disease (CKD) who were receiving hemodialysis.

Of the 101 patients enrolled, 97 yielded adequate transiliac bone biopsy specimens at baseline. Of the 22 patients whose iPTH level was within the KDOQI target range at the time of biopsy, only 2 had normal bone histology. Fourteen of the 22 patients had low-turnover bone disease, and 6 patients had high-turnover disease. After 1 year of treatment aimed at achieving the KDOQI targets for bone metabolism and disease in CKD, 64 patients provided a second biopsy sample. At this time point, the iPTH target was met by 16 patients, of whom only 1 had normal bone histology. Of the remaining 15 individuals, 1 had high-turnover bone disease and 14 had low-turnover disease. An iPTH level of $<150 \mathrm{ng} / \mathrm{l}$ had $50 \%$ sensitivity and $85 \%$ specificity for detection of low-turnover bone disease; an iPTH level of $>300 \mathrm{ng} / \mathrm{l}$ had $69 \%$ sensitivity and $75 \%$ specificity for diagnosis of high-turnover bone disease. 